Pacific Journal of Mathematic 


\title{
LIE ALGEBRAS OF LOCALLY COMPACT GROUPS
}

\author{
RICHARD K. LASHOF
}

1. Introduction. We call an LP-group, a group which is the projective limit of Lie groups. Yamabe [8] has proved that every connected locally compact group is an LP-group. This permits the extension to locally compact groups of the notion of a Lie algebra. In $\S \S 2$ and 3 we prove the existence and uniqueness of the Lie algebra of an LP-group and show the connection of the Lie algebra with the group by means of the exponential mapping.

In $\S 4$, we extend the notion of a universal covering group for connected groups with the same Lie algebra. A covering group of a connected group $g$, in the extended sense used here, means a pair $(\bar{g}, w)$, where $\bar{g}$ is a connected LP-group and $w$ is a continuous representation of $\bar{g}$ into $g$ which induces an isomorphism of the Lie algebra of $\bar{g}$ onto the Lie algebra of $g$ (see Definition 4.5). The universal covering group of a connected locally compact group is not necessarily locally compact and may not map onto the group. It turns out that the arc component of the identity in $\bar{g}$ is a covering space in the sense of Novosad [5] of the arc component of the identity of $g$ (these components are dense subgroups, Lemma 3.7).

Finally, in $\S 5$, we establish a one-to-one correspondence between " canonical LP-subgroups" of a group and subalgebras of its Lie algebra.

\section{Projective limit of Lie algebras.}

DEFINITION 2.1. By a topological Lie algebra (over the real numbers) we shall mean a (not necessarily finite dimensional) Lie algebra with an underlying topology such that the operations of addition, multiplication and scalar multiplication are continuous.

DeFinition 2.2. Let $J$ be an inductive set. Suppose given for each $a \in J$, a topological Lie algebra $G_{a}$ such that if $a<b$ there exists a continuous representation $f_{a b}: G_{b} \rightarrow G_{a}$. Let $G=\left[\left\{X_{a}\right\} \in \prod_{a \in J} G_{a}\right.$ such that $f_{a b} X_{b}=X_{a}$, all $a, b \in J$ with $\left.a<b\right]$. Then $G$ is a closed topological subalgebra of the direct product.

In analogy to A. Weil [7, p. 23], $G$ will be called the projective limit of the $G_{a}\left(G=\lim G_{a}\right)$ if the following hold:

Received September 27, 1955, and in revised form May 18, 1956. This paper is a portion of the author's doctoral dissertation, which was written at Columbia University. The author wishes to thank Professors Kadison and Taylor for their kind assistance. 
LP I: If $a<b<c, f_{a c} X_{c}=f_{a b}\left(f_{b c} X_{c}\right)$;

LP II: $f_{a b}$ is a continuous open homomorphism of $G_{b}$ onto $G_{a}$;

LP III: $f_{a}$, the natural projection of $G$ into $G_{a}$, is continuous and onto.

(Remark: $f_{a}$ open is implied by $f_{a b}$ open, (see [7, p. 24])

In particular, if the $G_{a}$ are finite dimensional Lie algebras with the usual topology as a Euclidean space, we get the following.

Theorem 2.3. Let $G_{a}$, $a \in J$, be a system of finite dimensional Lie algebras satisfying LP I and

LP II': $f_{a b}$ is a representation onto.

Then $G$ as defined in Definition 2.2 will necessarily satisfy LP II and LP III and hence $G=\lim G_{a}$.

Proof. LP II' implies LP II, since for finite dimensional vector spaces a representation onto is both continuous and open in the usual topology. That LP III is satisfied follows directly from the theory of linearly compact vector spaces [3 Ch. III, § 27]. In fact, this result holds for an inverse system of finite dimensional vector spaces.

Definition 2.4. If $G=\lim G_{a}, G_{a}$ finite dimensional Lie algebras, then $G$ will be called an LP algebra.

Lemma 2.5. Let $G=\lim G_{a}$, where the $G_{a}$ 's are complete topological Lie algebras, with homomorphisms $f_{a}$ and $f_{a b}$ satisfying LP I, II, and III. Let $N_{a}$ be the kernel of $f_{a}$, then
A. Every neighborhood of zero in $G$ contains an $N_{a}$
B. For each $N_{a}, N_{b}$; there exusts an $N_{c} \subset N_{a} \cap N_{b}$.
C. $G$ is complete.

Proof. It is easy to show (see [7]) that a fundamental system of neighborhoods of zero in $G$ is given by $f_{a}^{-1}\left(V_{a}\right), a \in J$, and $V_{a}$ running through a fundamental system of neighborhoods of zero in $G_{a}$. Condition A then follows directly.

If $c>a, b$ then $N_{c}$ obviously satisfies B. Condition $\mathrm{C}$ is immediate from the definition of $G$.

Lemma 2.6. Given a topological Lie algebra $G$ containing closed ideals $N_{a}$ satisfying A, B, C; then $\lim G / N_{a}$ exists and is isomorphic to $G$ (where we define $a<b$ if $N_{a} \supset N_{b}$ and let $f_{a b}: G / N_{b} \rightarrow G / N_{a}$ be the natural homomorphism). 
Proof. Since the Conditions A, B, C are identical to those for topological groups $\left[7\right.$, p. 25] it follows that $G$ is isomorphic to $\lim G / N_{a}$ as an additive topological group. The lemma now follows since the $N_{a}$ are ideals.

Theorem 2.7. Suppose $G=\lim G_{a}, G_{a}$ fiinite dimensional. If $K$ is a closed subalgebra of $G$, then $K=\lim K_{a}$, where $K_{a}$ is the image of $K$ in $G_{a}$. If $K$ is a closed ideal in $G$, then $G / K=\lim G_{a} / K_{a}$. In particular, $G / K$ is complete.

Proof. If $f_{a b}: G_{b} \rightarrow G_{a}(a<b)$, then $f_{a b}: K_{b} \rightarrow K_{a}$ satisfies LP I, II'. Hence $\lim K_{a}$ exists. Since $K$ maps onto $K_{a}, K$ is dense in $\lim K_{a}$, [7]. Since $K$ is closed, $K=\lim K_{a}$.

Likewise $G_{a} / K_{a}, a \in J$, satisfy LP I, II where $\bar{f}_{a b}: G_{b} / K_{b} \rightarrow G_{a} / K_{a}$ is induced by $f_{a b}$. Hence $\lim G_{a} / K_{a}$ exists. The natural maps $p_{a}: G_{a} \rightarrow$ $G_{a} / K_{a}$ evidently induce a map $p: G \rightarrow \lim G_{a} / K_{a}$ defined by: $p\left\{X_{a}\right\}=\left\{p_{a} X_{a}\right\}$, $\left\{X_{a}\right\} \in G$. This definition is legitimate since:

$$
\bar{f}_{a b}\left(p_{b} X_{b}\right)=p_{a}\left(f_{a b} X_{b}\right)=p_{a}\left(X_{a}\right), \quad a<b .
$$

This in turn induces a map $i: G / K \rightarrow \lim G_{a} / K_{a}$. We have to show that $i$ is an isomophism.

By its definition $i$ is evidently continuous and one-to-one into. We show that it is an isomorphism into. Since the natural map of $G$ onto $G / K$ is open, it is sufficient to show that if $W$ is a neighborhood in $G$ then $p(W)=p(W+K)$ is open. Now if $W$ is a neighborhood in $G$, take $V+V \subset W$. Then $V$ contains an $N_{a}$, kernel of $f_{a}: G \rightarrow G_{a}$ (Lemma 2.5). Then $p(W)$ contains $p(V)+p\left(N_{a}\right)$. Now $(p(V))_{a}=p_{a} f_{a}(V)$, is an open neighborhood in $G_{a} / K_{a}$; and the preimage in $\lim G_{a} / K_{a}$ of a neighborhood in $G_{a} / K_{a}$ is a neighborhood in $\because n G_{a} / K_{a}$. But if $(p(X))_{a} \in p_{a} f_{a}(V)$, $X \in G$ then $X_{a} \in f_{a}(V)+K_{a}$ and hence $X \in V+N_{a}+K \subset W+K$. But this implies that $i$ is open in $i(G / K)$ and hence is an isomorphism into.

It remains to show that $i$ is onto. But this follows since as an abstract vector space $G$ is linearly compact [3]. Hence

$$
\bigcap_{a \in J} f_{a}^{-1} p_{a}^{-1}\left(Y_{a}\right) \neq \phi, \quad\left\{Y_{a}\right\} \in \lim G_{a} / K_{a}
$$

since the intersection is nonempty for any finite subset of $J$.

LEMMA 2.8. Let $\theta$ be a continuous representation of an LP-algebra $G$ into an LP-algebra $H$. Then the image of $G$ in $H$ is a closed subalgebra of $H$.

Proof. Suppose $G=\lim G_{a}, a \in J ; H=\lim H_{b}, b \in K ; G_{a}$ and $H_{b}$ finite 
dimensional. Consider the map $p_{b}: G \rightarrow H_{b}$, composed of $\theta$ and the projection of $H$ onto $H_{b}$. This takes $G$ onto a subalgebra $H^{\prime}{ }_{b}$ of $H_{b}$. Obviously, the ${H^{\prime}}_{b}, b \in K$, define a closed subalgebra $H^{\prime}=\lim H^{\prime}{ }_{b}$ of $H$ under the induced system of representations. Also $\theta(G) \subset H^{\prime}$.

Let $Y=\left\{Y_{b}\right\}$ be in $H^{\prime}$. The preimage $p_{b}^{-1}\left(Y_{b}\right)$ is a closed linear variety in $G$. Since $G$ is linearly compact, $\cap p_{b}^{-1}\left(Y_{b}\right)$ is nonempty, as the intersection of a finite number is clearly nonempty. Hence there exists $X \in G$ such that $p_{b}(X)=Y_{b}$, all $b \in K$. Hence $G$ maps onto $H^{\prime}$. As $H^{\prime}$ is closed, this proves the Lemma.

LEMMA 2.9. Let $\theta$ be a continuous representation of an LP-algebra $G$ onto an LP-algebra $H$. Then $\theta$ is open.

Proof. Let $K$ be the kernel of $\theta$. By Theorem 2.7, $G / K$ is an LPalgebra. Hence it is clearly sufficient to prove the Lemma in the case that the map is also one-to-one.

Hence let $\theta$ be a continuous one-to-one representation of $G$ onto $H$. Suppose $G=\lim G / K_{a} ; K_{a}, a \leqslant J$, closed ideals in $G$ and $G / K_{a}$ finite dimensional. By Lemma 2.8, $\theta\left(K_{a}\right)$ is a closed ideal in $H$. Further $H / \theta\left(K_{a}\right)$ is finite dimensional and hence is (topologically) isomorphic to $G / K_{a}$. Since $\theta$ is continuous every neighborhood of $H$ contains some $\theta\left(K_{a}\right)$. It follows from Lemma 2.6 that

$$
H=\lim H / \theta\left(K_{a}\right)=\lim G / K_{a}=G .
$$

Clearly, the isomorphism so induced is the same map as $\theta$.

THEOREM 2.10. If $G_{1}$ and $G_{2}$ are two LP-algebras with the same underlying abstract algebra $G$, then $G_{1}$ and $G_{2}$ have the same topology.

Proof. By Lemma 2.9 it is sufficient to construct an LP-algebra $G_{0}$ whose underlying abstract algebra is $G$ and such that the identity maps of $G_{0}$ into $G_{1}$ and $G_{2}$ are both continuous. Let $K_{a}, a \in J$, be the set of all abstract ideals in $G$ which have finite codimension. As is well known, if $K_{a}$ and $K_{b}$ have finite codimension, so does $K_{a} \cap K_{b}$. It follows that $G / K_{a}, a \in J$, satisfy the conditions of Theorem 2.3, where we define $b>a$ if $K_{b} \subset K_{a}$ and $f_{a b}: G / K_{b} \rightarrow G / K_{a}$ is the natural projection. Let $G_{0}=\lim G / K_{a}$. We claim the underlying abstract algebra of $G_{0}$ is $G$.

Now $G$ is linearly compact since it is the underlying algebra of $G_{1}$ and $G_{2}$. Let $p_{a}: G \rightarrow G / K_{a}$ be the natural map. If $Y=\left\{Y_{a}\right\} \in G_{0}$, then $\cap p_{a}^{-1}\left(Y_{a}\right)$ is nonempty, since the intersection of a finite number is non-

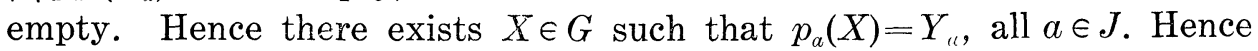
the map $p: X \rightarrow p_{a}(X)$ take $G$ onto $G_{0} . \quad p$ is one-to-one, since for every $X \in G$ there is a $K_{a}$ such that $p_{a}(X)$ is not zero, because the identity 
map of say $G$ onto $G_{1}$ is one-to-one.

Now $G_{1}=\lim G_{1} / K_{b}, b \in J^{\prime}, J^{\prime}$ a subset of $J . p^{-1}: G_{0} \rightarrow G_{1}$ is an isomorphism of this underlying abstract algebras and is continuous since $J^{\prime}$ is a subset of $J$. Hence the theorem follows by Lemma 2.9.

CoROLlary 2.11. Let $G$ be an abelian LP-algebra. Then $G$ is the direct product of 1-dimensional algehras. In particular, the underlying topological vector space of an LP-algebra is algebraically and topologically the direct product of 1-dimensional vector spaces.

\section{Lie algebra of an LP-group.}

DeFinition 3.1. Let $g_{\alpha}, a \in J$ be Lie groups. Suppose $g=\lim g_{a}$, the limit satisfying LP I, II, III of A. Weil [7]. Then we call $g$ an LP-group. (Note that $g$ is complete since the $g_{a}$ are complete.)

Definition 3.2. Suppose $g=\lim g_{a}, g_{a}$ connected Lie groups. Let $G_{a}$ be the Lie algebra of $g_{a}$. Then the homomorphisms $f_{a b}: g_{b} \rightarrow g_{a}(a<b)$ induce homomorphisms $d f_{a b}: G_{b} \rightarrow G_{a}$ satisfying LP I, II of Definition 2.1. Hence the $G_{a}, a \in J$, have a limit $G . G$ is called the Lie algebra of $g$.

We show in Lemma 3.4 below that $G$ is independent (in a natural sense) of the representation of $g$ as a limit of Lie groups.

DeFINITION 3.3. Suppose $g=\lim g_{a}, g_{a}$ connected Lie groups. Let $G, G=\lim G_{a}$, be the Lie algebra of $g$. Then we define a continuous map

$$
\exp : G \rightarrow g, \quad \exp \left\{X_{a}\right\}=\left\{\exp X_{a}\right\}, \quad\left\{X_{a}\right\} \in G .
$$

This mapping is legitimate, since if $f_{a b}: g_{b} \rightarrow g_{a}$ then

$$
f_{a b}\left(\exp X_{b}\right)=\exp d f_{a b} X_{b}=\exp X_{a}
$$

and hence $\left\{\exp X_{a}\right\} \in g$.

Lemma 3.4. Suppose $g=\lim g_{a}, g_{a}$ connected Lie groups, $a \in J ; h=$ $\lim h_{b}, h_{b}$ connected Lie groups, $b \in K$. Let $G=\lim G_{a}, H=\lim H_{b}$ be the corresponding Lie algebras. If $\theta: g \rightarrow h$ is an isomorphism then we can define an isomorphism $d \theta: G \rightarrow H$ such that

$$
\theta(\exp X)=\exp d \theta(X), \quad X \in G
$$

Proof. Let $f_{a}: g \rightarrow g_{a}$ and $\bar{f}_{b}: h \rightarrow h_{b}$ be the natural maps. Let $n_{a}$ and $\bar{n}_{b}$ be the kernels of $f_{a}$ and $\bar{f}_{b}$ respectively. Let $b \in K$. Since $h_{b}$ is a Lie group there is a neighborhood $V_{b}$ of $h_{b}$ which contains no non- 
trivial subgroups (that is, $h_{b}$ doesn't have arbitrarily small subgroups). Since $\bar{f}_{b} \theta: g \rightarrow h_{b}$ is continuous, there is a neighborhood $W$ in $g$ which maps into $V_{b}$. But $W$ contains some $n_{a}$ and this $n_{a}$ must go into the unit element of $h_{b}$. This defines a homomorphism $\theta_{b a}: g_{a} \rightarrow h_{b}$ such that

$$
\theta_{b a} f_{a}=\bar{f}_{b} \theta,
$$

this condition characterizing $\theta_{b a}$.

If $a>a^{\prime}, a$ and $a^{\prime}$ in $J$, then $f_{a}=f_{a a^{\prime}} f_{a^{\prime}}$ and $\theta_{b a} f_{a a^{\prime}} f_{t^{\prime}}=\bar{f}_{b} \theta$. Hence $\theta_{b a} f_{a a^{\prime}}=\theta_{b a^{\prime}}$. Similarly, if $b^{\prime}<b, \bar{f}_{b^{\prime} b} \theta_{b a}=\theta_{b^{\prime} u}$. The induced homomorphisms of the corresponding Lie algebras therefore satisfy

$$
d \theta_{b a} d f_{a a^{\prime}}=d \theta_{b a^{\prime}}, \quad d \bar{f}_{b^{\prime} b} d \theta_{b a}=d \theta_{b^{\prime} a} .
$$

It follows that the maps $d \theta_{b a}$ define a continuous representation $d \theta$ of $G$ into $H$, where $d \theta(X), X=\left\{X_{a}\right\} \in G$, is defined by

$$
(d \theta(X))_{b}=d \theta_{b a}\left(X_{a}\right) .
$$

This map is well defined because of the conditions satisfied above, and is continuous because $d \theta_{b a}$ is continuous.

Similarly for each $a \in J$, we can find a $b \in K$ and a homomorphism $\psi_{a b}: h_{b} \rightarrow g_{a}$ such that $f_{a} \theta^{-1}=\psi_{a b} \bar{f}_{b}$. This defines a continuous representation $d \psi$ of $H$ into $G$. Because of the conditions satisfied by the maps one sees easily that: $d \theta d \psi: H \rightarrow H$ and $d \psi d \theta: G \rightarrow G$ are the identities, and hence that $d \theta$ is an isomorphism.

Since $d \theta_{b a} d f_{a}=d \bar{f}_{b} d \theta$ by definition, we have

$$
\overline{f_{b}} \theta(\exp X)=\theta_{b a} f_{a}(\exp X)=\exp d \theta_{b a} d f_{a}(X)=\exp d \bar{f}_{a} d \theta(X) .
$$

By Definition 3.3, this implies that $\theta(\exp X)=\exp d \theta(X)$.

Theorem 3.5. Suppose $g=\lim g_{a}, g_{a}$ connected Lie groups. Let $G$ $=\lim G_{a}$ be the corresponding Lie algebra. Then $g$ is the closed subgroup generated by the elements of the form $\exp X, X \in G$.

Proof. Since $G$ maps onto $G_{a}$, $\exp X_{a}$ for $X \in G$ generates $g_{a}$, and $\exp G$ generates a dense subgroup of $g$, proving the theorem.

Lemma 3.6. Suppose $G=\lim G_{c}, G_{a}$ finite dimensional Lie algebras. Then the underlying space of $G$ is arcwise connected.

Proof. Since $G$ is a topological vector space it is arcwise connected by straight lines.

Lemma 3.7. Suppose $g=\lim g_{a}, g_{a}$ connected Lie groups. Then $g$ is 
connected and the arcwise connected component of $g$ is dense in $g$.

Proof. The map exp: $G \rightarrow g$ is continuous. Hence if $A$ is the image of $G, A$ is arcwise connected. Hence $A^{n}$ is arcwise connected. Hence $\bigcup_{1}^{\infty} A^{n}$ is arcwise connected. By Theorem 3.5 this is dense in $g$. Hence $g$ is connected.

THEOREM 3.8. Let $\left(g_{a}\right), a \in J$ be a system of connected Lie groups satisfying LP I, II of A. Weil [1]; then $g=\left(\left\{x_{a}\right\} \in \prod_{a \in J} G_{a} ; f_{a b} x_{b}=x_{a}\right.$, all $a, b \in J$ with $a<b)$ satisfies LP III, and hence:

$$
g=\lim g_{a}
$$

Proof. Let $G_{a}$ be the Lie algebra of $g_{a}$. Then the $\left(G_{a}\right), a \in J$ satisfy LP I, II of Definition 1.1. Hence they have a limit $G$. Let $X$ $\in G$, then if $X=\left\{X_{a}\right\}$ we have $\exp X_{a} \in g_{a}$ and $\left\{\exp X_{a}\right\} \in g$, since:

$$
f_{a b} \exp X_{b}=\exp d f_{a b} X_{b}=\exp X_{a}, a<b
$$

But elements of the form $\exp X_{a}$ generate $g_{a}$ since $G$ maps onto $G_{a}$. Hence $g$ maps onto $g_{a}$. Hence $g=\lim g_{a}$.

Lemma 3.9. Let $g=\lim g_{a}, g_{a}$ arbitrary Lie groups. Let $g_{a}^{0}$ be the connected component of the identity of $g_{a}$. Then the $\left(g_{a}^{0}\right), a \in J$ form $a$ system of groups satisfying LP I, II of $A$. Weil. Let $g^{0}=\lim g_{a}^{0}$, then $g^{0}$ is the connected component of $g$.

Proof. Since $f_{a b}: g_{b} \rightarrow g_{a}$ is continuous, open and $g_{b}^{0}$ is open in $g_{b}$, it takes $g_{b}^{0}$ onto $g_{a}^{0}$. Hence the $\left(g_{a}^{0}\right), a \in J$ satisfy LP I, II. By Theorem 3.8 they have a limit $g^{0} . \quad g^{0}$ may obviously be considered as a subgroup of $g$, closed since complete.

By Lemma 3.7, $g^{0}$ is connected and hence contained in the connected component of $g$. On the other hand, if $g_{1}$ is the connected component of $g, f_{a}\left(g_{1}\right)$ is connected and hence contained in $g_{a}^{0}$. Hence $g_{1}$ is contained in the limit of the $g_{a}^{0}$. Hence $g_{1}=g^{0}$.

Definition 3.10. Let $g$ be a topological group. If the connected component of the identity of $g$ is an LP-group, we define the Lie algebra of $g$ as the Lie algebra of its connected component.

REMARK. According to the result of Yamabe [8], every locally compact group is a generalized Lie group. This implies in particular that its connected component is an LP-group. Hence every locally com- 
pact group has a Lie algebra.

Theorem 3.11. Let $g$ and $h$ be topological groups for which Lie Algebras $G$ and $H$ are defined (Definition 3.10). Let $f$ be a continuous representation of $g$ into $h$, then $f$ induces a unique continuous representation of $G$ into $H$ such that $f(\exp X)=\exp d f(X), X \in G$.

Proof. Obviously $f$ defines a continuous representation of the connected component of $g$ into the connected component of $h$. Assume therefore that $g$ and $h$ are connected.

Suppose $g=\lim g_{a}, h=\lim h_{b}\left(g_{a}, h_{b}\right.$ connected Lie groups). The map $g \rightarrow h \rightarrow h_{b}$ induces a map of $g_{a} \rightarrow h_{b}$ for some $a$, since $h_{b}$ doesn't have arbitrarily small subgroups. This in turn induces a map of $G_{a} \rightarrow H_{b}$ and hence of $G$ into $H_{b}$. As in the proof of Lemma 3.4, it is easy to see that the maps $G \rightarrow H_{b}$ induce a continuous representation of $G$ into $H, d f: G \rightarrow H$; such that $f(\exp X)=\exp d f(X)$.

Suppose there are two such representations, say $d f$ and $\overline{d f}$. Then $(d f X)_{b} \neq(\overline{d f} X)_{b}$ some $b$ and $X$. Since any neighborhood of zero generates $G$, and since $d f$, $\overline{d f}$ are linear; we can chose $X$ such that $d f X$ and $\overline{d f} X$ are in any desired neighborhood of 0 in $H_{b}$. For a sufficiently small neighborhood exp is one-to-one on $H_{b}$. Hence $(\exp d f X)_{b}$ $\neq(\exp \overline{d f} X)_{b}$, a contradiction.

COROLlary. If $g$ is connected and $f, f_{1}$ are two representations of $g$ into $h$ such that $d f=d f_{1}$, then $f=f_{1}$.

Proof. Since $f(\exp X)=\exp d f X=\exp d f_{1} X=f_{1}(\exp X)$ and since $\exp G$ generates a dense subgroup of $g$, we have $f=f_{1}$.

LEMMA 3.12. Let $g$ and $h$ be locally compact topological group and $g$ connected, $G$ and $H$ their Lie algebras. Let $f$ be a continuous open homomorphism of $g$ onto $h$, then $d f$ is a continuous open homomorphism of $G$ onto $H$.

Proof. According to A. Weil [7], if $k$ is the kernel of $f$, we may take $g=\lim g_{a}, k=\lim k_{a}, k_{a}$ the image of $k$ in $g_{a}$, and $h=\lim g_{a} \mid k_{a}, g_{a}$ and $k_{a}$ Lie groups.

Then $G=\lim G_{a}, G_{a}$ the Lie algebra of $g_{a}$. Let $K$ be the Lie algebra of $k$; then $K=\lim K_{a}, K_{a}$ the Lie algera of $k_{a}$. Then the Lie algebra of $g_{a} / k_{a}$ is $G_{a} / K_{a}[1]$. Hence $H=\lim G_{a} / K_{a}$. By Theorem 2.5, $H=G / K$. It is easy to check that for $d f: G \rightarrow G / K$ that $f(\exp X)=\exp d f X$. So that the natural map of $G$ onto $G / K$ is $d f$. (Note: By a generaliza- 
tion of Pontrjagin's theorem on groups satisfying the second axiom of countability; if $f$ is continuous and onto it is automatically open.)

\section{Universal covering group.}

Definition 4.1. Suppose $g=\lim g_{a},\left(g_{a}\right), a \in J$ connected Lie groups. Let $\bar{g}_{a}$ be the simply connected covering group of $g_{a}$. The map $f_{a b}$ taking $g_{b}$ onto $g_{a}(a<b)$ induces an open homomorphism $\bar{f}_{a b}$ of $\bar{g}_{b}$ onto $\bar{g}_{a}$. Hence the $\left(\bar{g}_{a}\right), a \in J$ satisfy LP I, II and therefore have a limit $\bar{g}$ (Theorem 3.8). $\bar{g}$ is a complete, connected group. $\bar{g}$ is called the universal covering group of $g$.

Proposition 4.2. Let $G$ be the Lie algebra of $g$. Then $\bar{g}$ has the Lie algebra $G$, and there exists a continuous representation $w$ taking $\bar{g}$ onto a dense subgroup of $g$ such that $d w: G \rightarrow G$ is the identity.

Proof. The covering homomorphisms $w_{a}: \bar{g} \rightarrow g_{a}$ induce a continuous representation of $\bar{g}$ into $g$. Since ${ }^{d} w_{a}: G_{a} \rightarrow G_{a}$ is the identity, it follows that $d w$ is the identity. Since $w_{a}$ maps $\bar{g}_{a}$ onto $g_{a}$ it follows that $w(\bar{g})$ is dense in $g$.

Proposition 4.3. The kernel of $w$ is totally disconnected and is in the center.

Proof. If $k$ is the kernel of $w$, it follows from the definition of $w$ that the image $f_{c}(k)$ of $k$ in $g_{a}$ belongs to the kernel of $w_{a}$. But this kernel is discrete and hence $f_{a}(k)$ is discrete, and therefore closed in $g_{a}$. It follows that the $f_{a}(k)$ satisfy LP I, II and since $k$ is closed, $k=\lim f_{a}(k)$. Hence $k$ is the projective limit of discret groups. It follows from Lemma 3.9 that $k$ is totally disconnected. Further, a totally disconnected normal subgroup of a connected group belongs to the center.

LEMMA 4.4. Let $g$ be a connected LP-group with Lie algebra $G$. Let $h$ be any other connected LP-group with Lie algebra $H$ isomorphic to $G$. Then there exists an isomorphism of their universal covering groups $f: \bar{g} \rightarrow \bar{h}$ such that $d f$ is the given isomorphim of $G$ onto $H$.

Proof. Suppose $g=\lim g_{a}, h=\lim h_{b}$; then $\bar{h}=\lim \bar{h}_{b}, \bar{g}=\lim \bar{g}_{a}$ and $G=\lim G_{a}, H=\lim H_{b}$. The homomorphism $G \rightarrow H \rightarrow H_{b}$ induces a homomorphism $G_{a} \rightarrow H_{b}$ for some $a$, since $H_{b}$ has no small subgroups when considered as an additive group. This in turn induces a homomorphism of $\bar{g}_{a}$ onto $\bar{h}_{b}$. Similary there exist homomorphisms of $\bar{h}_{b}$ onto $\bar{g}_{a}, a \in J$, 
some $b$. As in the proof of Lemma 3.4, it is easy to see that this implies that there exists an isomorphism $f: \bar{g} \rightarrow \bar{h}$. The induced homomorphism $d f: G \rightarrow H$ such that $f(\exp X)=\exp d f(X)$, is obviously the original isomorphism of $G$ onto $H$.

Definition 4.5. Let $g$ and $h$ be connected LP-groups, $G$ and $H$ their Lie algebras. A continuous representation $w$ of $g$ into $h$ such that $d w$ is an isomorphism of $G$ onto $H$ is called a covering map and $g$ is called a covering group of $h,(g, w)$ is called the covering.

\section{Proposition 3.6. $w(g)$ is dense in $h$.}

Proof. In fact $w(\exp X)=\exp d w(X), X \in G$. But $\exp d w(X), X \in G$ generates a dense subgroup of $h$ since $d w$ is onto.

We now give a purely topological definition of covering space for arcwise connected spaces due to Novosad [5] and show that the arc component of the identity $g^{c}$ of $g$ in Definition 4.5 is actually a covering space in this sense of the arc component of the identity $h^{c}$ of $h$. (Note that $g^{c}$ is dense in $g$, Lemma 3.7) Similarly, we show the arc component of the identity of the universal covering group is a universal covering space.

Definition 4.7. (Novosad) Let $A$ be an arcwise connected space, $a \in A$. Let $f:(B, b) \rightarrow(A, a)$ be a continuous map of an arcwise connected space $B$ into $A$ taking $b$ into $a$. Then $(f, B, b)$ is called a covering space of $(A, a)$, if given any contractible space $C$, and point $c \in C$ which is a deformation retract of $C$, and a map $\alpha:(C, c) \rightarrow(A, c)$, then there exists a map $\bar{\alpha}:(C, c) \rightarrow(B, b)$ which is unique with respect to the property $f \bar{\alpha}=\alpha$.

Let $\left(P_{A}, a \#\right)$ be the pair consisting of the space of paths starting from $a \in A$, with the compact open topology, of an arbitrary topological space $A$, and the constant path $a \#$ at $a \in A$. A continuous map $f:(B, b)$ $\rightarrow(A, a)$ induces a continuous map $f \sharp:\left(P_{B}, b \sharp\right) \rightarrow\left(P_{A}, a \sharp\right)$ defined by:

$$
f \sharp(p)(t)=f(p(t)), p \in P_{B}, t \in I \text { (the unit interval) }
$$

It is then easy to see that Definition 4.7 is equivalent to the following.

Definition $4.7^{\prime}$. Let $f:(B, b) \rightarrow(A, a)$ be a continuous map of an arcwise connected space $B$ into an arcwise connected space $A$ taking $b \in B$ into $a \in A$. Then $(f, B, b)$ is called a covering space of $(A, a)$ if $f \sharp:\left(P_{B}, b \sharp\right) \rightarrow\left(P_{A}, a \#\right)$ is a homeomorphism onto. 
Proposition 4.8. Definitions 4.7 and $4.7^{\prime}$ are equivalent.

Proof.

(4.7) implies $\left(4.7^{\prime}\right)$ by Lemma 2.3 of [5].

(4.7') implies (4.7); since, let $\lambda_{A}: P_{A} \rightarrow A, \lambda_{A}(p)=p(1), p \in P_{A}$. Then $\lambda_{A}$ defines a fiber space (in the sense of Serre) which obviously satisfies the covering homotopy theorem for arbitrary spaces. Hence if $\alpha:(C, c)$ $\rightarrow(A, a)$ is homotopic to tne constant map the homotopy may be lifted to $\left(P_{A}, a \sharp\right)$ and hence to $\left(P_{B}, b \sharp\right)$ by the homeomorphism of $\left(4.7^{\prime}\right) . \lambda_{B}$ maps the image into $(B, b)$. The endpoint of the homotopy gives the desired covering of (4.7). The uniquenes follows since any point of $C$ describes a path under the retraction and the image of this path in $B$ is unique since covering paths are unique by $\left(4.7^{\prime}\right)$.

Definition 4.9. An arcwise connected space $A$ is called simply connected if every covering space $(4.7)$ of $(A, a)$ is trivial. This property is independent of the base point $a \in A$ (see [5]).

Let $\Omega_{A}$ be the (closed) subspace of $P_{A}$ consisting of closed paths (that is, the loop space). Let $\Omega_{A}^{c}$ be the arc component of $a \#$ in $\Omega_{A}$.

Theorem 4.10. Let $A$ be an arcwise and locally arcwise connected space, $a \in A$; then if $\Omega_{A}$ is connected (not necessarily arcwise connected) and $\Omega_{A}^{c}$ is dense in $\Omega_{A}, A$ is simply connected (Definition 4.9).

Proof. Let $(f, B, b)$ be a covering space of $(A, a)$. Then $f \sharp: P_{B}$ $\rightarrow P_{A}$ is a homeomorphism, and hence $f \#$ maps $\Omega_{B}$ homeomorphically into $\Omega_{A}$. But every loop in $A$ contractible to $a$ may be lifted to a unique loop in $B$ (see proof of 4.8), hence $f \sharp\left(\Omega_{B}\right) \supset \Omega_{A}^{c}$. But $f \sharp\left(\Omega_{B}\right)$ is closed in $P_{A}$. Hence $f \#$ maps $\Omega_{B}$ onto $\Omega_{A}$.

Now this means that $f: B \rightarrow A$ is one-to-one. For if $f \sharp(p)(1)=f \sharp\left(p^{\prime}\right)$ .(1), $p, p^{\prime} \in P_{B}$, then $p(1)=p^{\prime}(1)$; since $f \sharp p$ and $f \sharp p^{\prime}$ having the some endpoint form a loop in $A$, and hence must come from a loop in $B$ by the above. Also $p(1)=p^{\prime}(1)$ implies $(f \sharp p)(1)=\left(f \sharp p^{\prime}\right)(1)$. Hence since $\lambda_{B}$ : $P_{B} \rightarrow B$, and $\lambda_{A}: P_{A} \rightarrow A$ (the endpoint maps) are onto, $f$ is one-to-one.

Further $\lambda_{A}$ is both continuous and open [4, Lemma 4] since $A$ is locally arcwise connected, hence the continuous map $\lambda_{B} f^{-1}: P_{A} \rightarrow B$ induces a continuous map $f^{-1}: A \rightarrow B$. This proves the theorem.

We now apply the above to LP-groups. We remark that if $g$ is a topological group; then $P_{g}$, the space of paths of $g$ beginning at the identity, may be made into a topological group by pointwise multiplication of paths. Then $\Omega_{g}$ is a closed normal subgroup.

Lemma 4,11. Let $g=\lim g_{\alpha}, g_{\alpha}$ Lie groups; then $P_{g}=\lim P_{a_{\alpha}}$. 
Proof. First $f_{a}: g \rightarrow g_{a}$ has a local cross-section. In fact, it is obvious that $d f_{a}: G \rightarrow G_{a}$ has a cross-section since these spaces are linear, further $g_{a}$ has a neighborhood which is homeomorphic to a neighborhood of $G_{a}$ and exp: $G \rightarrow g$ is continuous.

Now $P_{g_{a}}$ is arcwise connected, hence $f_{a}^{\sharp}: P_{g} \rightarrow P_{g_{a}}$ will be onto if the image covers a neighborhood of the identity. But this follows from the local cross-section of $g_{a}$ in $g$. In fact, a fundamental system of neighborhoods of the identity in $P_{a}$ is obtained from a fundamental system of neighborhoods of the identity in $g$ by taking all those paths that are contained in a given neighborhood of the identity in $g$. Also it follows that $f_{a}^{\#}$ is open.

If $V$ is a neighborhood of the identity in $g$ that contains the kernel $k_{a}$ of $f_{a}$, then the corresponding neighborhood in $P_{g}$ contains $P_{k_{a}}$, and this last is clearly the kernel of $f_{a}^{\sharp}$. Finally, $P_{g}$ is complete since $g$ is complete. Hence all the conditions for a projective limit are satisfied and the lemma follows.

LEMmA 4.12. If $g=\lim g_{a}, g_{n}$ simply connected Lie groups, then $\Omega_{g}$ $=\lim \Omega_{q_{l i}}$.

Pooof. The proof is the same as above, using the fact that since $g_{a}$ is simply connected, $\Omega_{g_{a}}$ is arcwise connected.

LeMmA 4.13. If $g=\lim g_{a}, g_{a}$ simply connected, then $\Omega_{g}^{c}$ is dense in $\Omega_{g}$ and $\Omega_{g}$ is connected.

Proof. Since $f_{a}: g \rightarrow g_{a}$ has local cross-sections (see 4.11 ), it defines a principal fiber bundle and hence satisfies the covering homotopy theorem of [6]. Hence since every loop in $g_{a}$ is contractible each loop may be lifted to a contractible loop in $g$. Hence $\Omega_{g}^{c}$ maps onto $\Omega_{a_{a}}$, all $a$. Hence $\Omega_{g}^{c}$ is dense in $\Omega_{g}$. The last statement then follows.

Lemma 4.14. If $g=\lim g_{a}, g_{a}$ simply connected, then $P_{g} / \Omega \approx g^{c}$.

Proof. For Lie groups, $P_{g_{a}} / \Omega_{g_{a}} \approx g_{a}$, since $g_{a}$ is locally arcwise connected and hence the map of $P_{g_{a}}$ onto $g_{a}$ is open (see [7]). Since $P_{g}$ maps continuously onto $P_{g_{a}}, P_{g}$ maps continuously onto $P_{g_{a}} / \Omega_{g_{a}} \approx g_{a}$. The induced map of $P_{g}$ into $g=\lim g_{a}$ is obviously $\lambda_{g}$, and this induces a continuous one-to-one map of $P_{g} / \Omega_{g}$ onto $g^{c} \subset g$. Hence it is sufficient to show that $P_{g} / \Omega_{g}$ has the proper topology as a subgroup of $g$.

The neighborhoods of the identity in $P_{g} / \Omega_{g}$ are of the form $V \Omega_{g}$, where $V$ is the preimage in $P_{g}$ of a neighborhood $V_{a}$ in $P_{g_{a}}$. But this is the preimage in $P_{g} / \Omega_{g}$ of the neighborhood $V_{a} \Omega_{g_{a}}$ in $P_{g_{a}} / \Omega_{g_{a}} \approx g_{a}$. Hence the Lemma follows. 
THEOREM 4.15. If $g=\lim g_{a}, g_{a}$ simply connected Lie groups, then $g^{c}$ (the arc-component of the identity) is arcwise connected, locally arcwise connected, and simply connected (Definition 4.9).

Proof. $g^{c}$ is locally arcwise connected since $P_{g}$ is obviously so. Hence by Lemmas 4.12 and 4.13 and Theorem $4.10, g^{c}$ is simply connected.

COROLlaRY 4.16. If $g$ is the universal covering group (4.1) of $a$ metrisable LP-group or a connected locally compact group, then $g$ is arcwise connected, locally arcwise connected and simply connected.

Proof. For metrisable groups, $P_{g}$ is metrisable and complete. Hence $P_{g} / \Omega_{g} \approx g^{c}$ is complete and thus $g^{c}=g$. The result for locally compact groups will follow from Theorem 4.25.

We write again $w$ for the map of Definition 4.5 cut down to $g^{c}$.

Lemma 4.17. $\left(w, g^{c}, e\right)$ is a covering space (Definition 4.7) of $\left(h^{c}, e\right)$.

Proof. First assume $g=\bar{h}$ the universal covering group of $h$. Then if $h=\lim h_{a}, \bar{h}=\lim \bar{h}_{a}$ and since $P_{\bar{h}_{a}} \approx P_{h_{a}}$ (this is obvious for Lie groups), $P_{\bar{h}} \approx \lim P_{\bar{h}_{a}} \approx \lim P_{h_{a}} \approx P_{h}$. Further, this isomorphism is clearly induced by the covering map $w$.

Now let $(g, w)$ be any covering group of $h$. Then $\bar{h} \approx \bar{g}$ by (4.4) and hence $P_{g} \simeq P_{\bar{g}} \approx P_{\bar{h}} \approx P_{h}$. Since $\bar{g} \rightarrow g \rightarrow h$ is the same as $\bar{g} \rightarrow \bar{h} \rightarrow h$. The isomorphism $P_{g} \approx P_{h}$ is induced by $w$. Hence $\left(w, g^{c}, e\right)$ satisfies $(4.7)^{\prime}$.

THEOREM 4.18. If $g$ and $h$ are LP-groups, $G$ and $H$ their Lie algebras, $\bar{g}$ and $\bar{h}$ their universal covering groups, $P_{g}$ and $P_{h}$ their group of paths, $\Omega_{g}^{c}$ and $\Omega_{h}^{c}$ the arc component of the identity in their group of loops, respectively; then the following are equivalent:
(a) $G$ isomorphic to $H$
(b) $\bar{g}$ isomorphic to $\bar{h}$
(c) $P_{g}$ isomorphic to $P_{\text {. }}$ such that the isomorphism takes $\Omega_{g}^{c}$ onto $\Omega_{h}^{c}$.

Proof.

(a) implies (b) follows from (4.4).

(b) implies (a) follows from (4.2).

(b) implies (c): $P_{g} \simeq P_{\bar{g}}$ by (4.17). Also $\Omega_{\sigma}^{c} \simeq \Omega_{g}^{c}$ under the same 
map since every contractible loop in $g$ may be lifted to $\bar{g}$ (see proof of 4.8).

(c) implies (b): writing $\bar{\Omega}_{g}^{c}$ for the closure of $\Omega_{g}^{c}$ in $P_{g}$, we have

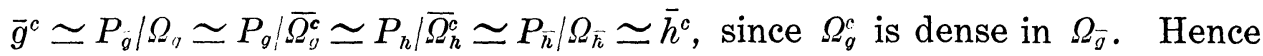
$\bar{g} \simeq \bar{h}$.

LEMMA 4.19. Every LP-algebra is the Lie algebra of an LP-group.

Proof. By assumption, if $G$ is an LP-algebra then $G=\lim G_{a}, G_{a}$ finite dimensional. Let $g_{a}$ be the simply connected groups corresponding to $G_{a}$. The homomorphisms of $G_{a}$ onto $G_{b}(a<b)$, induce homomorphisms of $g_{a}$ onto $g_{b}$ which satisfy LP I, II. Hence they have a limit $g$ (Theorem 3.8). But $g$ obviously has Lie algebra $G$.

Definition 4.20. The group $g$ defined in the proof of (4.19) is called the universal group corresponding to $G$.

LEMma 4.21. Let $g$ be a universal LP-group then every covering group $(h, w)$ (Definition 4.5) is trivial, that is, $w$ is an isomorphism of $h$ onto $g$.

Proof. $h^{c}$ is a trivial covering of $g^{c}$. Since $h$ and $g$ are complete and $h^{c}$ and $g^{c}$ are dense, the lemma follows.

THEOREM 4.22. Let $h$ be an LP-group and let $H$ be its Lie algebra. Let $G$ be an LP-algebra and $g$ the universal group corresponding to $G$. Let $\theta$ be a continuous representation of $G$ into $H$. Then $\theta$ induces a continuous representation $f$ of $g$ into $h$ such that $d f=\theta$.

Proof. If $h=\lim h_{b}, h_{b}$ Lie groups; then $H=\lim H_{b}, H_{b}$ finite dimensional Lie algebras. Suppose $G=\lim G_{a}, G_{a}$ finite dimensional. Let $g_{a}$ be the simply connected group corresponding to $G_{a}, g=\lim g_{a}$. The map $G \rightarrow H \rightarrow H_{b}$ induces a map $G_{a} \rightarrow H_{b}$, some $a$. But this in turn induces a map $g_{a} \rightarrow h_{b}$ and hence a map of $g \rightarrow h_{b}$ for every $b$. It is easy to see that this defines a map $f: g \rightarrow h$ such that $d f=\theta$.

THEOREM 4.23. The universal covering group $\bar{g}$ of a connected locally compact group $g$ is the direct product of simply connected Lie groups. More explicitly $\bar{g} \simeq h \times a \times s$, where $h$ is a simply connected Lie group, $a$ is the (possibly infinite) direct product of the reals and $s$ is the (possibly infinite) direct product of simple simply connected compact Lie groups, 
Proof. According to Yamabe [8] and Iwasawa (Theorem 11 of [2]), $g$ is locally the direct product of a local Lie group $h^{\prime}$ and a compact normal subgroup $k$. Now $k=\lim k_{a}$, where $k_{a}=k / n_{a}, n_{a}$ normal in $k$ and hence in $g$ (Theorem 4 of [2]). Hence $g=\lim g_{a}$, where $g_{a}=g / n_{a}$. Evidently $g_{a}$ is locally isomorphic to $h^{\prime} \times k_{a}$; and hence to $h^{\prime} \times k_{a}^{0}, k_{a}^{0}$ connected component of $k_{a}$ (since $k_{a}$ a Lie group).

Since $f_{a b}: g_{b} \rightarrow g_{a}$ takes $k_{b}$ onto $k_{a}$, it takes $k_{b}^{0}$ onto $k_{a}^{0}$. Hence $f_{a b}$ induces a homomorphism of $h \times \bar{k}_{b}^{0}$ onto $h \times \bar{k}_{a}^{0}$, where $h$ is the simply connected group associated with $h^{\prime}$ and $\overrightarrow{k_{a}^{0}}$ is the simply connected covering group of $k_{a}^{0}$. If $\bar{k}^{0}=\lim \bar{k}_{a}^{0}$, then $k \times \bar{k}^{0}=\lim h \times \bar{k}_{a}^{0} ; h \times \bar{k}_{a}^{0}$ is the simply connected covering group of $g_{10}$ and hence $h \times \bar{k}^{0}$ is the universal covering group of $g$.

Since $k^{0}$ is the universal covering group of $k^{0}, k^{0}$ connected component of $k$; the problem is reduced to considering the universal covering group of a compact connected group.

According to A. Weil (p. 91 of [7]), $k^{0}$ is isomorphic to $\left(a^{\prime} \times s\right) / d$, where $a^{\prime}$ is a compact abelian connected group, $s$ is the (possibly infinite) direct product of simple simply connected compact Lie groups, and $d$ is totally disconnected. It is evident that $\bar{k}^{0}=a \times s$, where $a$ is the universal covering group of $a^{\prime}$. Since $a^{\prime}$ in the projective limit of toroidal groups, $a$ is the projective limit of vector groups, and hence is the direct product of the reals (2.11). This proves the theorem.

COROLlaRY 4.24. If $g$ is a locally compact group, then its Lie algebra $G$ has the form $G=H \times A \times S$, where $H$ is a finite dimensional Lie algebra, $A$ is the product (possibly infinite) of 1-dimensional Lie algebras, and $S$ is the (possibly infinite) direct product of simple compact Lie algebras.

EXAmple 1. Let $g=\| l T_{a}, a \in J, T_{a}$ isomorphic to the torus group, all $a$. Then $g$ is compact. But $\bar{g}=\Pi R_{a}, R_{a}$ isomorphic to the additive group of reals, $a \in J$. Hence for $J$ infinite, $\bar{g}$ is not locally compact.

Example 2. Let $P$ be the $p$-adic solenoid (See for example: Eilenberg and Steenrod, Foundations of algebraic topology, p. 230). $P$ is a compact connected group and is the projective limit of torus groups. If $T$ is the multiplicative group of all complex numbers $z$ with $|z|=1$, the projections $\varphi: T \rightarrow T$ are given by $\varphi(z)=z^{p}, p$ an integer. $\varphi$ induces the map $\bar{\varphi}: R \rightarrow R, \bar{\varphi}(x)=p x$, which is an isomorphism of the additive group of the reals onto itself. Hence the universal covering group of $P$, which is the projective limit of the reals under these isomorphisms, is itself the additive group of reals. Hence the Lie algebra of $P$ is 1 - 
dimensional. As is well known (see above reference) $R$ maps continuously, one-to-one onto a dense subgroup of $P$, not the whole group. The map is not open and not onto $P$. More generally we have the following.

EXAMPLE 3. Let $g$ be a connected, but not locally connected, locally compact group. Let $(\bar{g}, w)$ be its universal covering group. Then $w: \bar{g} \rightarrow g$ is not both open onto. Consequently, if $\bar{g}$ is locally compact, $w$ is neither open (on the image) nor onto.

In fact $\bar{g}$ is locally connected. Hence if $w$ is open and onto, $g$ would be locally connected. If $\bar{g}$ is locally compact then if $w$ is open on $w(\bar{g})$, $w(\bar{g})$ would be locally compact, hence closed, hence $w(\bar{g})=g$. On the other hand, if $w$ is onto and $\bar{g}$ connected locally compact, $w$ is open.

Hence, in particular we have the following. Let $g$ be a connected, but not locally connected finite dimensional locally compact group. Then $\bar{g}$ is locally compact (a Lie group) and hence $w$ is neither open nor onto.

EXAMPLE 4. Not every complete topological Lie algebra is an LPalgebra. In fact an infinite dimensional Banach space cannot contain arbitrarily small subspaces and cannot be an abelian LP-algebra.

\section{Subgroups and subalgebras.}

DeFINITION 5.1. Let $g$ be an LP-group. An LP-group $h$ is called an LP-subgroup of $g$ if $h$ is an abstract subgroup and the inclusion map $f: h \rightarrow g$ is a continuous representation such that $d f: H \rightarrow G$ is an isomorphism into.

TheOREM 5.2. Let $g=\lim g_{a}$ be a LP-group; $G=\lim G_{a}$ its Lie algebra. Let $H$ be a closed subalgebra of $G$, then $H=\lim H_{a}$ where $H_{a}$ is the image of $H$ in $G_{a}$. Let $h_{a}$ be the analytic subgroup of $g_{a}$ corresponding to $H_{a}$. Then $h=\lim h_{a}$ exists and is a connected LP-subgroup of $g$ with Lie algebra $H$.

Proof. $\quad H=\lim H_{a}$ follows from Theorem 2.5. Now $f_{a b}: g_{b} \rightarrow g_{a}$ induces $\bar{f}_{a b}: h_{b} \rightarrow h_{a}$, onto since the image of $h_{b}$ in $g_{a}$ is the analytic subgroup of $g_{a}$ whose Lie algebra is $H_{a}$ [1]. Therefore the $h_{a}$ satisfy LP I, II and hence have a limit $h$ (Theorem 3.8).

Obviously $h$ is an abstract subgroup of $g$ and the maps $h_{a} \rightarrow g_{a}$ induce a continuous one-to-one representation of $h$ into $g$, namely the inclusion map. Obviously $d f$ is the inclusion map of $H$ into $G$ and hence an isomorphism into. 
Lemma 5.3. Let $h$ be the LP-subgroup of $g$ defined in Theorem 5.2. Let $h^{\prime}$ be any other connected LP-subgroup with Lie algebra $H^{\prime}$ and such that the inclusion map $f^{\prime}: h^{\prime} \rightarrow g$ induces an isomorphism $d f^{\prime}$ of $H^{\prime}$ onto $H$. Then $h^{\prime}$ is a covering group of $h$ and the covering map is abstractly an inclusion.

Proof. Suppose $h^{\prime}=\lim h_{b}^{\prime}$, $h_{b}^{\prime}$ Lie groups. The map $h^{\prime} \rightarrow g \rightarrow g_{a}$ induces a map $h_{b}^{\prime} \rightarrow g_{a}$, for some $b$. But the image of $h_{b}^{\prime}$ in $g_{a}$ is the analytic subgroup whose Lie algebra is $H_{a}$, the image of $H_{b}^{\prime}$ in $G_{a}$. Hence, $h_{b}^{\prime} \rightarrow h_{a}$ is continuous and open, and induces $h^{\prime} \rightarrow h_{a}$ continuous, open. This induces a continuous representation $\theta: h^{\prime} \rightarrow h$ such that $d \theta$ is the isomorphism $d g^{\prime}$. Since $h^{\prime}$ is contained abstractly in $g$, its elements are determined by their coordinates in $g_{a}$. Hence $\theta$ is an abstract inclusion of $h^{\prime}$ in $h$.

CoRollary. The subgroup $h$ defined in Theorem 5.2 is uniquely characterized by Lemma 5.3.

Proof. Suppose $h$ and $h^{\prime}$ are two connected LP-subgroups such that $h$ is a covering group of $h^{\prime}$ and $h^{\prime}$ is a covering group of $h$, and such that the covering maps are abstract inclusions. Then the maps $h \rightarrow h^{\prime} \rightarrow h$ and $h^{\prime} \rightarrow h \rightarrow h^{\prime}$ are both the identity. Hence $h=h^{\prime}$.

Definition 5.4. The LP-subgroup $h$ of $g$ defined in Theorem 5.2 is called the canonical LP-subgroup corresponding to the subalgebra $H$ of $G$.

We have proved the following.

Theorem 5.5. Let $g$ be an LP-group, $G$ its Lie algebra. There exists a one to one correspondence between canonical PL-subgroups of $g$ and closed subalgebras of $G$.

THEOREM 5.6. Let $g$ be the universal group corresponding to an LPalgebra $G$. Let $k$ be a closed normal connected subgroup of $g$. Then $k$ is the canonical LP-subgroup corresponding to an ideal in $G$. Conversely, the canonical LP-subgroup corresponding to an ideal in $G$ is a closed normal connected topological subgroup of $g$.

Proof. Let $k$ be a closed, normal, connected subgroup of $g$. Suppose $g=\lim g_{a}, g_{a}$ simply connected Lie groups. Let the image of $k$ in $g_{a}$ be $k_{a}$. The closure $\bar{k}_{a}$ of $k_{a}$ in $g_{a}$ is a closed connected normal subgroup of $g_{a}$. Let $K_{a}$ be the ideal of $G_{a}$ corresponding to $\bar{k}_{a}$.

The image by $f_{a b}$ of $\bar{k}_{b}$ in $g_{a}(a<b)$, is the analytic subgroup of $g_{a}$ corresponding to the image of $K_{b}$ in $G_{a}$. But the image is an ideal in 
$G_{a}$, hence the corresponding analytic subgroup is a closed normal connected subgroup of $g_{a}$. Hence $\bar{k}_{a} \subset f_{a b}\left(\bar{k}_{b}\right)$. On the other hand since $f_{a b}$ is continuous, $f_{a b}\left(\bar{k}_{b}\right) \subset \overline{k_{a}}$. Hence $f_{a b}\left(\bar{k}_{b}\right)=\bar{k}_{a}$.

Hence $k^{\prime}=\lim \bar{k}_{a}$ exists and is a closed normal subgroup of $g$, and is the canonical $L P$-subgroup corresponding to $K=\lim K_{a}$. On the other hand, $k<K^{\prime}$; but $k$ maps onto $k_{a}$, a dense subgroup of $\vec{k}_{a}$. Hence $k$ is dense in $k^{\prime}$ and $k=k^{\prime}$ since $k$ is closed.

The converse is obvious since the analytic subgroups of $g_{a}$ corresponding to ideals are closed topological subgroups.

ExAmple. Consider the $p$-adic solenoid of Example 2, $\S 4$. The additive group of reals $R$ may be considered as an LP-subgroup of the $p$-adic solenoid $P$. Then $P$ itself is the canonical LP-subgroup corresponding to the Lie algebra of $R$.

\section{REFERENCES}

1. C. Chevalley, Theory of Lie groups, Vol. 1, Princeton, 1946.

2. K. Iwasawa, On some types of topological groups, Ann. of Math., 50 (1949), 507-558.

3. S. Lefschetz, Algebric topology, Amer. Math. Soc. Colloq. Pub., no. 27, 1942.

4. J. Moore, Introduction to homotopy theory, Mimeographed Notes, Princeton Univ., 1955.

5. R. S. Novosad, Simply connected spaces, Trans. Amer. Math. Soc., 79 (1955), 216-228.

6. N. Steenrod, The topology of fibre bundles, Princeton, 1951.

7. A. Weil, L'Integration dans les groupes topologique, 2nd ed., Hermann \& Cie. Paris, 1953.

8. H. Yamabe, A generalization of a theorem of Gleason, Ann. of Math., 58 (1953), 351-365. 


\section{PACIFIC JOURNAL OF MATHEMATICS}

\section{EDITORS}

H. L. ROYDEN

Stanford University

Stanford, California

R. A. Beaumont

University of Washington

Seattle 5, Washington

\section{A. L. Whiteman}

University of Southern California

Los Angeles 7, California

E. G. Straus

University of California

Los Angeles 24, California

\section{ASSOCIATE EDITORS}
E. F. BECKENBACH
C. E. BURGESS
M. HALL
E. HEWITT

\author{
A. HORN \\ V. GANAPATHY IYER \\ R. D. JAMES \\ M. S. KNEBELMAN
}

L. NACHBIN

I. NIVEN

G. SZEKERES

T. G. OSTROM

M. M. SCHIFFER
F. WOLF

K. YOSIDA

\section{SUPPORTING INSTITUTIONS}

\author{
UNIVERSITY OF BRITISH COLUMBIA \\ CALIFORNIA INSTITUTE OF TECHNOLOGY \\ UNIVERSITY OF CALIFORNIA \\ MONTANA STATE UNIVERSITY \\ UNIVERSITY OF NEVADA \\ OREGON STATE COLLEGE \\ UNIVERSITY OF OREGON \\ UNIVERSITY OF SOUTHERN CALIFORNIA
}

\author{
STANFORD UNIVERSITY \\ UNIVERSITY OF UTAH \\ WASHINGTON STATE COLLEGE \\ UNIVERSITY OF WASHINGTON \\ AMERICAN MATHEMATICAL SOCIETY \\ CALIFORNIA RESEARCH CORPORATION \\ HUGHES AIRCRAFT COMPANY \\ THE RAMO-WOOLDRIDGE CORPORATION
}

Mathematical papers intended for publication in the Pacific Journal of Mathematics should be typewritten (double spaced), and the author should keep a complete copy. Manuscripts may be sent to any of the editors. All other communications to the editors should be addressed to the managing editor, E. G. Straus at the University of California, Los Angeles 24, California.

50 reprints per author of each article are furnished free of charge; additional copies may be obtained at cost in multiples of 50 .

The Pacific Journal, of Mathematics is published quarterly, in March, June, September, and December. The price per volume (4 numbers) is $\$ 12.00$; single issues, $\$ 3.50$. Back numbers are available. Special price to individual faculty members of supporting institutions and to individual members of the American Mathematical Society: $\$ 4.00$ per volume; single issues, $\$ 1.25$.

Subscriptions, orders for back numbers, and changes of address should be sent to Pacific Journal of Mathematics, 2120 Oxford Street, Berkeley 4, California.

Printed at Kokusai Bunken Insatsusha (International Academic Printing Co., Ltd.), No. 10, 1-chome, Fujimi-cho, Chiyoda-ku, Tokyo, Japan.

PUBLISHED BY PACIFIC JOURNAL OF MATHEMATICS, A NON-PROFIT CORPORATION

The Supporting Institutions listed above contribute to the cost of publication of this Journal, but they are not owners or publishers and have no responsibility for its content or policies. 


\section{Pacific Journal of Mathematics}

\section{Vol. 7, No. 2 \\ February, 1957}

William F. Donoghue, Jr., The lattice of invariant subspaces of a completely continuous quasi-nilpotent transformation ................... 1031

Michael (Mihály) Fekete and J. L. Walsh, Asymptotic behavior of restricted extremal polynomials and of their zeros.................... 1037

Shaul Foguel, Biorthogonal systems in Banach spaces ............... 1065

David Gale, A theorem on flows in networks ................... 1073

Ioan M. James, On spaces with a multiplication .................. 1083

Richard Vincent Kadison and Isadore Manual Singer, Three test problems in operator theory .................................... 1101

Maurice Kennedy, A convergence theorem for a certain class of Markoff processes........................................ 1107

G. Kurepa, On a new reciprocity, distribution and duality law ........ 1125

Richard Kenneth Lashof, Lie algebras of locally compact groups ........ 1145

Calvin T. Long, Note on normal numbers .................... 1163

M. Mikolás, On certain sums generating the Dedekind sums and their reciprocity laws ..................................... 1167

Barrett O'Neill, Induced homology homomorphisms for set-valued maps......................................... 1179

Mary Ellen Rudin, A topological characterization of sets of real numbers........................................... 1185

M. Schiffer, The Fredholm eigen values of plane domains 1187

F. A. Valentine, A three point convexity property .........

Alexander Doniphan Wallace, The center of a compact lattice is totally

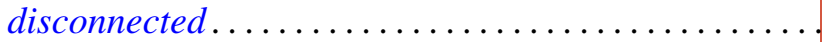

Alexander Doniphan Wallace, Two theorems on topological lattices.

G. T. Whyburn, Dimension and non-density preservation of mappings...

John Hunter Williamson, On the functional representation of certain algebraic systems ... 